\title{
The Discovery of Druggable Anti-aging Agents
}

\author{
Gulperi Yalcin, Cheol-Koo Lee \\ Department of Biotechnology, College of Life Sciences and Biotechnology, Korea University, Seoul, Korea
}

Corresponding Author:

Cheol-Koo Lee, $\mathrm{PhD}$

Department of Biotechnology, College

of Life Sciences and Biotechnology,

Korea University, Seoul 02841, Korea

E-mail: cklee2005@korea.ac.kr

ORCID:

https://orcid.org/0000-0003-3927-9195

Received: December 9, 2020

Accepted: December 10, 2020
Caloric restriction (CR) has been shown to extend the lifespan of many species. Research to identify compounds that imitate the results of CR has shown extensions of both lifespan and healthspan via different mechanisms. For example, mechanistic target of rapamycin (mTOR) inhibitors such as rapamycin affect nutrient-sensing pathways; phenols and flavonoids show antioxidant characteristics; aspirin has anti-inflammatory effects, while spermidine induces autophagy. Herein, we summarize research progress and proposed mechanisms for the most well-known compounds showing lifespan-extending potential for anti-aging characteristics.

Key Words: Caloric restriction mimetics, Anti-aging, Lifespan extension

\section{INTRODUCTION}

While humanity's interest in defeating mortality is ancient, recent studies on anti-aging biology have been shaped after the first discovery in 1939 of retarded growth and a longer lifespan in rats with restricted caloric intake. ${ }^{1)}$ Since then, caloric restriction (CR) has been proven to be the most effective method for lifespan extension, with consistent reports from independent research groups in species including microbes, worms, fish, rodents, ${ }^{2,3)}$ and monkeys. ${ }^{4)}$ Similar to CR, another dietary method, methionine restriction, has also consistently shown promising results. ${ }^{5,6)}$ However, despite these positive results, researchers have also searched for chemicals, so-called caloric restriction mimetics (CRMs) that replicate these results without requiring restrictive diets.

Research on CRMs to identify anti-aging chemicals has shown that chemicals with potential lifespan-extending effects function through diverse pathways. Because CR is a good reference point, its resulting phenotype could facilitate the search for anti-aging agents. In the context of outcomes, CR extends lifespan by ameliorating several aging hallmarks such as DNA damage, accumulation of reactive oxygen species (ROS), insulin resistance, stress response, and the occurrence of cancer. ${ }^{7)}$ The exact mechanisms by which CR induces these shifts in aging parameters are not yet completely elucidated; however, several mechanisms have been suggested, most of which are conserved across different species. One such mechanism is via nutrient receptor pathways. Through these nutrient receptors, the lack of nutrients prevents the activity of several mechanisms that play a role in growth and biosynthesis and instead activates pathways that promote repair, autophagy, and maintenance. Indeed, CR inhibits both insulin/insulin-like growth factor-I (IGF-I) signaling ${ }^{8)}$ and key regulators in its downstream phosphatidylinositol-3 kinase (PI3K) and mechanistic target of rapamycin (mTOR) pathways. ${ }^{9)}$ Inhibition of the mTOR pathway blocks protein synthesis and other anabolic pathways and simultaneously promotes autophagy. ${ }^{10)}$ Therefore, the inhibition of the insulin/IGF-I and mTOR signaling pathways could be useful in the search for potential anti-aging agents.

Another way that lifespan may be improved is through the prevention of oxidative damage. ROS are important causes of aging, as free radicals produced through respiratory and metabolic pathways accumulate over time and damage chromosomal and mitochondrial DNA, which deteriorate mitochondrial function alongside other cellular mechanisms. This damage causes the eventual malfunction of cells, which might lead to cancer, along with other age-related conditions. ${ }^{11)} \mathrm{CR}$ activates nuclear factor (erythroidderived 2)-like 2 ( Nrf2), a regulator of antioxidants and other cytoprotective enzymes. ${ }^{12)}$

Sirtuins have also been suggested to play a key role in lifespan 
extension. Sirtuins are a nicotinamide adenine dinucleotide (NA$\mathrm{D}^{+}$)-dependent protein class of ADP-ribosyltransferases and deacetylases. They are activated by an increased $\mathrm{NAD}^{+} / \mathrm{NADH}$ ratio, which occurs during starvation or low energy status. Upon activation, sirtuins regulate stress response mechanisms such as antioxidant expression, anti-inflammatory proteins, autophagy, and DNA repair. ${ }^{13)}$ Hence, CR has been proposed to provide benefits mainly through sirtuin activation, especially that of Sir2 (SIRT1 in mammals), the overexpression of which extended lifespan in yeast. ${ }^{14)}$

Another approach to lifespan extension focuses on inducing low-level stress that does not damage the organism but rather activates stress response pathways. This approach predicts that $\mathrm{CR}$ does not elongate lifespan due to the overall reduction in nutrient intake, but rather due to the longer periods of starvation between meals. Cells can detect starvation in several ways. During low energy status, cells try to increase the amount of ATP with a reaction that uses two ADPs and releases AMP as a byproduct. This increase in AMP levels activates AMP-activated protein kinase (AMPK). AMPK is a master regulator of metabolism and activates catabolic pathways while suppressing anabolic pathways to balance energy homeostasis. AMPK was reportedly activated during CR. ${ }^{15)}$ AMPK activation can ensure cell survival through the stimulation of the oxidative stress response and autophagy, making it another indicator in anti-aging chemical studies, similar to sirtuins.

One of methods for identifying CRMs was to examine how they affect the activity levels of certain transcription factors. Among these factors is $\mathrm{FoxO}$, which can induce antioxidant expression and promote autophagy and mitophagy. FoxO activity is regulated by $\mathrm{PI} 3 \mathrm{~K} / \mathrm{AKT} / \mathrm{mTOR}$, AMPK, and sirtuins, making it a critical target during energy level shifts that would occur in certain diets. ${ }^{13)} \mathrm{In}$ addition, nuclear factor- $\mathrm{kB}$ (NF-kB) may also play a critical role in the impact of $\mathrm{CR}$ on lifespan. Aging is associated with increased levels of chronic inflammation and $\mathrm{CR}$ has been found to inhibit NF- $\mathrm{kB}$ and its proinflammatory effect. ${ }^{16)}$ Furthermore, NF- $\mathrm{kB}$ activity can be regulated by SIRT $1 .{ }^{17)}$

Finally, all key pathways affected by CR induce the deacetylation of certain cellular proteins and activate autophagy. Therefore, according to Madeo et al., ${ }^{18)}$ all CRMs should have the capacity to induce autophagy through protein deacetylation using at least one of the CR targets. Indeed, many of the widely accepted and potential CRMs decrease protein acetylation levels and increase autophagy. $^{19)}$

The most straightforward method of determining whether a chemical can increase lifespan is to expose the subject for long periods and have the result at the very end, which can be affected by other factors. Therefore, the following footprints of certain mecha- nisms, such as CR, help identify potentially life-extending chemicals. Such indicators include nutrient receptor pathways such as insulin/IGF-I and mTOR signaling, antioxidant and autophagy activity, deacetylation levels, or transcription factors such as FoxO, $\mathrm{Nrf2}$, and NF- $\mathrm{kB}$ as well as levels of certain genes and byproducts that are major regulators of potentially lifespan-extending mechanisms, including sirtuins, $\mathrm{NAD}^{+}$, and AMPK. However, a chemical can activate one of these pathways and have no eventual effect on lifespan or could increase the lifespan without showing an association with these pathways, as not every factor related to aging has yet been discovered. This review introduces some of the most powerful candidates for extending lifespan.

\section{THE MOST STUDIED POTENTIAL ANTI-AGING COMPOUNDS}

\section{Resveratrol}

Among potential CRMs, one of the most studied is resveratrol. This non-flavonoid natural phenol is found in many plants, especially grapes. Studies showed that resveratrol treatment induced a transcriptional pattern parallel to that of CR in mice. ${ }^{20)}$ Functionally, resveratrol can induce effects similar to those of $\mathrm{CR}$, including AMPK activation and increased SIRT1 levels in humans, ${ }^{21)}$ as well as autophagy induction, ${ }^{22)}$ and inhibition of NF-kB-mediated cytokine expression. ${ }^{23)}$ Resveratrol also delays age-associated parameters in mice in a way that partially mimicked CR. ${ }^{24)}$ Nevertheless, despite its anti-aging characteristics, including a positive effect on oxidative stress and even possible cancer-prevention effects, ${ }^{25)}$ resveratrol did not show the expected results on increasing lifespan. While resveratrol extended the lifespan in some vertebrates ${ }^{26)}$ and flies, ${ }^{27)}$ this effect was not observed in most studies, including those in yeast ${ }^{28)}$ and mice. ${ }^{20)}$ In addition, while resveratrol reversed insulin resistance caused by obesity, diabetes, or a high-fat diet, it failed to increase insulin sensitivity in healthy individuals. ${ }^{29)}$ Moreover, the positive results are conditional. As a supplement in older mice fed a high-protein diet, resveratrol showed adverse effects, instead increasing inflammation and ROS production. ${ }^{30)}$ Due to its promising potential, resveratrol has been the subject of many clinical trials for patients with diabetes, obesity, or problems with cognitive function. The latest results suggested that, despite inducing similar gene expression to that for $\mathrm{CR}$ and possibly improving mitochondrial number and function, resveratrol might not have significant benefits on glucose metabolism in such patients. ${ }^{31,32)}$

\section{Sirtuin Activators and $\mathrm{NAD}^{+}$Boosters}

Sirtuin activation is a signature of CR; thus, any sirtuin activator is also a potential CRM candidate. SRT1720 and SRT2104 are both 
synthetically produced SIRT1 activators that have shown great anti-aging effects. SRT1720 increased insulin sensitivity and improved both health and lifespan alongside reduced age-related risk factors in obese and standard diet-fed mice. ${ }^{33,34)}$ SRT2104 also showed similar effects, with increased mean and maximal lifespan and improved bone mineral density and insulin sensitivity in mice fed with standard diet. ${ }^{35)}$ More recently, SRT1720 reduced smoking-induced oxidative stress and mitochondrial dysfunction by activating SIRT1 in human alveolar epithelial cells. ${ }^{36)}$ Such chemicals require further investigation for use as anti-aging compounds; however, due to their sirtuin-activating characteristics, they are being used in various studies.

The $\mathrm{NAD}^{+} / \mathrm{NADH}$ ratio within cells directly regulates sirtuin activity; therefore, precursors or intermediates that increase $\mathrm{NAD}^{+}$ concentration are an alternative to direct sirtuin activators. CR upregulates NADH-dehydrogenases expressed by $\mathrm{Nqo} 1$ and $\mathrm{Cyb5r3}$ genes in rodents. The overexpression of these metabolic genes to imitate CR benefits showed reduced chronic inflammation, improved physical performance, and protection against cancer, with a mild lifespan increase. ${ }^{37)} \mathrm{NAD}^{+}$itself is important for mitochondrial activity. In mice, its precursor, nicotinamide riboside (NR), delayed senescence in neural stem cells and increased lifespan. ${ }^{38)} \mathrm{NR}$ has also been the subject of clinical trials; its chronic supplementation increased $\mathrm{NAD}^{+}$levels and reduce inflammatory cytokine levels in the skeletal muscle of older people. ${ }^{39,40)}$ Other precursors, including nicotinamide (NAM) and nicotinamide mononucleotide (NMN), reduced oxidative stress, improved insulin sensitivity, and suppressed inflammation in obese mice fed a high-fat diet. ${ }^{41,42)}$ However, despite having anti-aging effects, these two compounds did not extend the overall lifespan. A recent study in Caenorhabditis elegans and human cell cultures suggested that longevity can be increased by nicotinic acid (NA) supplementation only when the intracellular $\mathrm{NAD}^{+}$level is lower than the sirtuin-saturating concentration, thereby limiting its benefits to individuals with lower intracellular $\mathrm{NAD}^{+43)}$ These conditions might also apply to other $\mathrm{NAD}^{+}$-boosting chemicals.

\section{Polyphenols/antioxidants}

Polyphenols and their subspecies flavonoids are another group of chemicals that have attracted attention for their anti-aging capabilities. These compounds are obtained from plants and are known mainly for their antioxidant characteristics. Compounds such as quercetin, tangeretin, and catechins in tea have been studied for their effects, including anti-oxidative stress resistance and anti-inflammation. Quercetin was shown to activate the antioxidant Nrf2 pathway, which is also a target for CR. ${ }^{44)}$ However, the benefits of these compounds are not limited to oxidative stress prevention.

\section{Quercetin}

Quercetin reduced the acetylation of cytoplasmic proteins, which promoted autophagy ${ }^{45)}$ and also reduced endothelial oxidative injury via SIRT 1 activation. ${ }^{46)}$ Some of the other reported benefits have been more controversial. In primary human adipocytes, quercetin inhibited TNF- $\alpha-$ mediated inflammation and insulin resistance as potently as resveratrol; ${ }^{47)}$ however, in a clinical trial, it showed no impact on insulin resistance or other cardiovascular risk factors. ${ }^{48)}$ Similarly, quercetin increased the lifespan in yeast ${ }^{49)}$ and C. elegans ${ }^{50)}$ through a mechanism independent of the FoxO transcription factor. However, quercetin also reportedly did not affect the lifespans of flies or mice. ${ }^{51,52)}$ Another study showed that quercetin in combination with dasatinib promoted the healthspan of mice; in this study, these drugs showed a senolytic effect, which can be used as a tissue rejuvenation technique to selectively eliminate senescent cells through the activation of apoptotic pathways. ${ }^{53)}$ Similarly, the combination of quercetin and dasatinib was administered in a clinical study of patients with cellular senescence-associated lung disease, idiopathic pulmonary fibrosis (IPF) showed that this combination decreased the physical dysfunction caused by IPF. ${ }^{54)}$ These findings suggest the possible anti-aging effects of quercetin through mechanisms distinct from those of CR.

\section{Caffeic acid}

Caffeic acid is a natural phenolic antioxidant. This molecule affects many of the pathways in the anti-aging checklist. For example, caffeic acid induced deacetylation and autophagy; ${ }^{45}$ its effect on the mitochondrial respiratory chain to ameliorate oxidative injury was connected to SIRT3 activity. ${ }^{55)}$ It activated AMPK in multiple cancer cell lines, also. ${ }^{56)}$ Some of its derivatives, including caffeic acid phenethyl ester (CAPE), have been suggested as anti-cancer agents because they induce apoptosis in both human colon cancer and cervical carcinoma cells. In a mouse model, this activity was shown to occur through PI3K/AKT, AMPK, and mTOR signaling cascades in both in vitro and in vivo experiments. ${ }^{57)}$ Although it is known as an antioxidant molecule, elevated ROS and oxidative stress induced by CAPE triggered apoptosis in a human cervical carcinoma cell line. ${ }^{58)}$ In addition to its anti-carcinogen effects, CAPE inhibited neuroinflammation and motor neuron cell death, while increasing the survival of a mouse model of amyotrophic lateral sclerosis. ${ }^{59)}$ Both caffeic acid and CAPE have been shown to extend the lifespan of C. elegans. ${ }^{60,61)}$ In addition, caffeic acid increased the lifespan of fruit flies with reduced oxidative damage and increased stress resistance. ${ }^{62)}$ Interestingly, CAPE seems to trigger different pathways in different species, as its activity depends on the DAF-16 (FoxO homolog) and not on SKN-1 (Nrf2 homolog) in C. elegans, whereas it showed the opposite pattern in 
a mammalian cell line by activating $\mathrm{Nrf} 2$ and not FoxO4 signaling. ${ }^{61)}$

\section{Curcumin}

Curcumin is another polyphenol example of a CRM candidate. It is the major component of turmeric in Indian curry. Curcumin activates AMPK signaling, induces autophagy, ${ }^{63)}$ inhibits the PI3K/ $\mathrm{AKT} / \mathrm{mTOR}$ signaling pathway, ${ }^{64)}$ and suppresses NF- $\mathrm{kB}$-mediated inflammation. ${ }^{23)}$ It has also been shown to improve insulin resistance in rats. ${ }^{65)}$ Curcumin extended the lifespan in flies ${ }^{66)}$ but not in mice. ${ }^{52)}$ While its biometabolite tetrahydrocurcumin may be more effective, resulted in an extended mean lifespan in both mice and fruit flies, ${ }^{67,68)}$ curcumin continues to be the subject of hundreds of clinical trials.

Patients with chronic kidney disease are prone to an early loss of muscle and bone mass and are, therefore, regarded as a state of premature aging. This damage is proposed to be partly caused by high oxidative damage. Curcumin in combination with resveratrol alleviated this damage and helped to significantly increase the muscle and bone mass of patients after 12 weeks of supplementation. ${ }^{69)}$ In healthy middle-aged and older people, curcumin improved resistance artery endothelial function by reducing oxidative stress, although it did not affect arterial stiffness. ${ }^{70)}$

\section{Other phenols}

Antioxidants are widely accepted as anti-aging compounds, and resveratrol, also a natural phenol; quercetin; $\mathrm{CAPE}$; and curcumin have all been shown to modulate, increase, or ameliorate the activity of major antioxidant enzymes such as superoxide dismutase (SOD), catalase (CAT), and glutathione peroxidase (GPx). ${ }^{71-74)}$ However, these effects are mostly observable under certain conditions and after cells are exposed to a stress factor. In CR, cells exhibit higher activity or gene expression related to antioxidant enzymes upon exposure to stress. ${ }^{75,76)}$ However, in the absence of stress, i.e., under normal conditions, MnSOD and $\mathrm{Cu} / \mathrm{Zn}-\mathrm{SOD}$ activities were significantly lower in rats on a moderate CR diet $(20 \%-40 \%)$ than in control group rats. ${ }^{77)}$ This might be because CR indirectly reduces ROS by slowing pro-aging and growth pathways or by activating mitophagy so that antioxidant activity is not needed. In addition, it is not completely clear whether antioxidant supplementation is always good for health as ROS might also play biological roles. For instance, vitamin $\mathrm{C}$, also known as ascorbic acid, is a well-known antioxidant that has been shown to increase lifespan in yeast. ${ }^{78)}$ However, in pharmacologic concentrations, it acted as a pro-oxidant to reduce aggressive tumor growth in mice, similar to the results of studies of CAPE in human cervical carcinoma. ${ }^{58,79)}$ Therefore, the benefits of these natural phenols and flavo- noids go beyond their antioxidant activity and involve several different pathways. As another example, flavonoid 4,4-dimethoxychalcone from the Japanese ashitaba plant was shown to increase the lifespan of yeast cells by activating autophagy through the inhibition of Gln3, independent of the mTOR pathway. ${ }^{80)}$

\section{Other Important Anti-aging Compounds}

\section{Aspirin}

Aspirin, also known as acetylsalicylic acid, is an anti-inflammatory drug that is also considered a CR mimetic. Aspirin has been shown to extend the lifespans of $C$. elegans, fruit flies, and mice. ${ }^{81-83)}$ In $C$. elegans, aspirin reduced ROS levels, increased antioxidant gene expression, and was suggested to function through FoxO transcription factor activity. ${ }^{81)}$ In another similar study, aspirin was shown to act through the AMPK pathway independent of SIR-2.1. ${ }^{84)}$ In murine tumor cells, aspirin inhibited mTOR and promoted autophagy to suppress tumor growth. ${ }^{85)}$ Therefore, overall, studies with aspirin are quite promising. In a mouse study, life extension was only observed in male mice, suggesting differences in drug metabolism between sexes. ${ }^{83)}$ Aspirin is currently used by humans for many reasons and has attracted attention, especially after it was shown to reduce cardiovascular disease (CVD)-related mortality when was used as secondary prevention, i.e., in patients with a previous a cardiovascular event. ${ }^{86,87)}$ Aspirin was also assessed as a primary preventative method for heart disease and further for any mortality risk. The Aspirin in Reducing Events in the Elderly (ASPREE) trial was a study that started collecting data in 2010 and scheduled to finish by 2024 . This study included over 19,000 older adults with no known disorder who consumed a low dosage of daily aspirin. Unfortunately, the results were not as expected, since it showed not only no difference in CVD-related mortality but also increased hemorrhage and all-cause mortality in subjects, causing the early termination of data collection. ${ }^{88-90)}$

\section{Spermidine}

The activation of autophagy is an important CRM characteristic. In this context, spermidine is a well-known compound among autophagy-inducing agents. Spermidine is a polyamine that extended the lifespan of several species, including yeast, C. elegans, and mice in an autophagy-dependent manner. ${ }^{22,91)}$ In mice, it boosted cardiac autophagy and mitochondrial respiration by inhibiting oxidative stress. In addition, observational showed lower blood pressure and a lower incidence of cardiovascular disease in people with higher dietary consumption of spermidine. ${ }^{91)}$ While spermidine activates AMPK and inhibits mTOR, ${ }^{92)}$ its mechanism differs from that of resveratrol; thus, these two compounds could synergistically in- 
duce autophagy. ${ }^{22)}$ In clinical studies, spermidine was well-tolerated and improved memory performance in older adults with cognitive decline, making it a good target for cognitive aging studies. ${ }^{93,94)}$

\section{Metformin}

The antiglycation agent metformin is another potential life-extending chemical. It is normally used for the treatment of diabetes and high blood pressure and is also considered a CRM, as it also shows multiple CR-like effects. Metformin improved glucose homeostasis, reduced oxidative damage, inhibited mTOR, and activated AMPK signaling, through which it also upregulated SIRT1 expression and induced autophagy. ${ }^{95-97)}$ Metformin has been reported to extend lifespan in yeast, C. elegans, and mice. ${ }^{97-99)}$ Its life-extending capability in C. elegans was dependent on SKN-1 (Nrf2) antioxidant activity alongside AMPK signaling. ${ }^{99)}$ While most of the metformin clinical studies targeted diabetic patients, metformin also reduced levels of aging-associated cytokines in both diabetic and non-diabetic older adults ${ }^{100)}$ and had also been suggested as an anti-cancer drug, as it inhibited pro-oncogenic pathways and tumor growth. $96,101,102)$

\section{Rapamycin}

The mechanism by which the mTOR inhibitor rapamycin directly binds to mTOR complex 1 is well known and the reason for which this antifungal chemical was named as mTOR. mTOR is a fundamental regulator of cell growth and proliferation and is evolutionarily preserved across species. ${ }^{103)}$ Rapamycin extends the lifespan of various species, including yeast, fruit flies, and mice. ${ }^{28,104,105)}$ While it has also been suggested as a CRM, some of the metabolic changes induced by $\mathrm{CR}$ were absent in rapamycin-treated mice, in addition to having different patterns of gene expression related to xenobiotic metabolism in the liver. ${ }^{106)}$ Rapamycin was also shown to have a distinct impact on energy metabolism compared to $\mathrm{CR},{ }^{107)}$ which was followed by a report that it induces a shift in amino acid metabolism. ${ }^{108)}$

The life-extending characteristic of rapamycin is promising. Rapamycin treatment increased the median lifespan in mice in a dose- and sex-dependent manner, where higher doses and female sex showed a higher-percentage increase in longevity. ${ }^{106)}$ Rapamycin's ability to increase lifespan may be related to its growth-suppressing effects. Rapamycin is currently used as an immunosuppressant to avoid graft rejection in organ transplantation. ${ }^{109)}$ On top of suppressing the immune system, rapamycin may have other effects. For instance, rapamycin increased mortality in mice with type-2 diabetes, ${ }^{110)}$ while chronic treatment with rapamycin negatively affected glucose tolerance, causing insulin resistance. This effect was not observed in mice with genetically reduced mTOR ex- pression, suggesting that rapamycin caused this insulin resistance independent of mTORC1 activity. ${ }^{111)}$

Due to its precedence as a competent compound, rapamycin analogs such as everolimus and other mTOR inhibitors such as Torin $1 / 2$ are also being studied for their anti-aging characteristics. ${ }^{112,113)}$ Everolimus has been reported to reverse age-induced gene expression in old rat kidneys ${ }^{114)}$ and to improve cognitive function in a mouse Alzheimer's model. ${ }^{115)}$

\section{Senolytics}

Aged or senescent cells can release factors into the bloodstream or extracellular matrix to trigger younger and healthier cells to enter a senescent state. Thus, within an organism, rather than trying to reverse aging within every cell, the selective elimination of senescent cells has been hypothesized to boost overall youth in the tissue. To achieve such results, a variety of methods are currently being developed. The programmed death of senescent cells has been shown to improve the function of several tissues and increase the median lifespan in mice. ${ }^{116)}$ Senolytics are a class of chemicals that can trigger senescent cell apoptosis. Some examples of senolytic chemicals are quercetin and dasatinib, which are kinase inhibitors, and 17-DMAG, which is a heat shock protein inhibitor. 17-DMAG improved the healthspan of mice with progeroid syndrome ${ }^{117)}$ and, the combination of dasatinib and quercetin increased the health and median lifespan of both progeroid and naturally aging mice. $^{53,118)}$ As mentioned previously, the use of quercetin and dasatinib significantly improved the physical capability of people with cellular senescence-associated lung disease, although pulmonary function itself was unchanged. ${ }^{54)}$ Based on these results, senolytic drugs have attracted attention in current studies on tissue rejuvenation.

\section{Ketone Bodies}

Other than CR, many diets and fasting methods benefit metabolism. In most of these, the target is to activate pathways to increase autophagy, ketogenesis, stress resistance, and other protective mechanisms. Unlike other supplement-based methods, the ketogenic diet $(\mathrm{KD})$ changes the ratio of the nutrients consumed and elevates ketone body levels within the body more naturally. Without changing the overall calorie consumption, by minimizing carbohydrate intake and replacing it with protein and fat, the $\mathrm{KD}$ can increase circulating ketone body levels and fatty acid oxidation to mimic starvation metabolism. In mice, KD inhibited mTOR signaling and reduced the cancer incidence, which increased the median lifespan. ${ }^{119)}$ However, the results in glucoregulatory mechanisms are controversial as they have been shown to improve insulin/glucose sensitivity and cause glucose intolerance. ${ }^{119,120)}$ In hu- 
mans, the $\mathrm{KD}$ improved weight loss and reduced blood pressure compared to a low-fat diet. ${ }^{121)}$

\section{CONCLUSION}

The discovery of life-extending chemicals is difficult, as the metabolisms causing the aging phenotype within a cell or whole organism have not been fully elucidated. While many potential life-extending chemicals have been studied for their ability to induce CRlike metabolic changes, these are not merely less potent imitators or $\mathrm{CR}$, as many can stimulate mechanisms that are not included in the regulation of conventional nutrient-related pathways. For instance, quercetin showed senolytic effects, unlike most CRMs, while many antioxidants were more potent in activating multiple SOD or catalase pathways than CR. ${ }^{122)}$ These differences, even if they are not necessarily bad for metabolism, may be overlooked in the search for anti-aging chemicals, as most research focuses on the known pathways for CR. These overlooked pathways might be the main reason for anti-aging effects and not because they mimicked any of the major metabolic shifts induced by CR.

Although some are also considered CRMs, antioxidants, in general, are one of the largest groups of chemicals that are considered anti-aging. Their benefits, however, are controversial as their effects are often condition-dependent. In fact, many antioxidants are reported to be both anti- and pro-aging. For instance, a C. elegans study showed that vitamin $\mathrm{C}$ only had life-extending characteristics for certain knockout backgrounds with altered ROS levels and showed no clear effects on the wild-type strain. Moreover, antioxidant $\mathrm{N}$-acetylcysteine (NAC) and vitamin $\mathrm{C}$ were both shown to improve and damage lifespan, depending on the dose level of the supplement and innate ROS levels in the strain. ${ }^{123)}$

Based on the current capacity of anti-aging chemicals, rather than identifying the perfect compound, it might be crucial to instead focus on two or three chemicals that can function better when combined. Such combinations may stimulate more pathways when they are complementary to each other or reduce side effects when more efficiently targeting the same pathways. Some of these potential CRM systems, such as resveratrol and spermidine, have been tested as combination treatments and have been shown to work synergistically. ${ }^{22)}$

Finally, besides identifying the most effective anti-aging chemicals, it might be essential to combine these supplements with compatible diets and other lifestyle changes, such as exercise, to achieve significant differences. As there is no universal solution for every age, gender, or health condition, it is important to study and compare different aspects of these discoveries.

\section{ACKNOWLEDGMENTS}

\section{CONFLICT OF INTEREST}

The researchers claim no conflicts of interest.

\section{FUNDING}

This work was supported by National Research Foundation (NRF, Republic of Korea) grants funded by the Korean government (No. NRF-2020R1F1A1075785).

\section{AUTHOR CONTRIBUTION}

Conceptualization, CKL; Writing-original draft, GY, CKL; Writing-review \& editing, GY, CKL.

\section{REFERENCES}

1. McCay CM, Maynard LA, Sperling G, Barnes LL. Retarded growth, life span, ultimate body size and age changes in the albino rat after feeding diets restricted in calories: four figures.J Nutr 1939;18:1-13.

2. Weindruch R, Walford RL. The retardation of aging and disease by dietary restriction. Springfield, IL: C. C. Thomas; 1988.

3. Koubova J, Guarente L. How does calorie restriction work? Genes Dev 2003;17:313-21.

4. Colman RJ, Anderson RM, Johnson SC, Kastman EK, Kosmatka KJ, Beasley TM, et al. Caloric restriction delays disease onset and mortality in rhesus monkeys. Science 2009;325:201-4.

5. Lee BC, Kaya A, Ma S, Kim G, Gerashchenko MV, Yim SH, et al. Methionine restriction extends lifespan of Drosophila melanogaster under conditions of low amino-acid status. Nat Commun 2014;5:3592.

6. Ruckenstuhl C, Netzberger C, Entfellner I, Carmona-Gutierrez D, Kickenweiz T, Stekovic S, et al. Lifespan extension by methionine restriction requires autophagy-dependent vacuolar acidification. PLoS Genet 2014;10:e1004347.

7. Daniel M, Tollefsbol TO. Epigenetic linkage of aging, cancer and nutrition. J Exp Biol 2015;218(Pt 1):59-70.

8. Fontana L, Villareal DT, Das SK, Smith SR, Meydani SN, Pittas AG, et al. Effects of 2-year calorie restriction on circulating levels of IGF-1, IGF-binding proteins and cortisol in nonobese men and women: a randomized clinical trial. Aging Cell 2016;15:227.

9. Blagosklonny MV. Calorie restriction: decelerating mTOR-driven aging from cells to organisms (including humans). Cell Cycle 2010;9:683-8.

10. Jung CH, Ro SH, Cao J, Otto NM, Kim DH. mTOR regulation of autophagy. FEBS Lett 2010;584:1287-95. 
11. Santos AL, Sinha S, Lindner AB. The good, the bad, and the ugly of ROS: new insights on aging and aging-related diseases from eukaryotic and prokaryotic model organisms. Oxid Med Cell Longev 2018;2018:1941285.

12. Martin-Montalvo A, Villalba JM, Navas P, de Cabo R. NRF2, cancer and calorie restriction. Oncogene 2011;30:505-20.

13. Oellerich MF, Potente M. FOXOs and sirtuins in vascular growth, maintenance, and aging. Circ Res 2012;110:1238-51.

14. Kaeberlein M, McVey M, Guarente L. The SIR2/3/4 complex and SIR2 alone promote longevity in Saccharomyces cerevisiae by two different mechanisms. Genes Dev 1999;13:2570-80.

15. Canto C, Auwerx J. Calorie restriction: is AMPK a key sensor and effector? Physiology (Bethesda) 2011;26:214-24.

16. Jung KJ, Lee EK, Kim JY, Zou Y, Sung B, Heo HS, et al. Effect of short term calorie restriction on pro-inflammatory NF-kB and AP-1 in aged rat kidney. Inflamm Res 2009;58:143-50.

17. Yeung F, Hoberg JE, Ramsey CS, Keller MD, Jones DR, Frye RA, et al. Modulation of NF-kappaB-dependent transcription and cell survival by the SIRT1 deacetylase. EMBO J 2004;23: 2369-80.

18. Madeo F, Carmona-Gutierrez D, Hofer SJ, Kroemer G. Caloric restriction mimetics against age-associated disease: targets, mechanisms, and therapeutic potential. Cell Metab 2019; 29:592-610.

19. Martens CR, Seals DR. Practical alternatives to chronic caloric restriction for optimizing vascular function with ageing. J Physiol 2016;594:7177-95.

20. Pearson KJ, Baur JA, Lewis KN, Peshkin L, Price NL, Labinskyy $\mathrm{N}$, et al. Resveratrol delays age-related deterioration and mimics transcriptional aspects of dietary restriction without extending life span. Cell Metab 2008;8:157-68.

21. Timmers S, Konings E, Bilet L, Houtkooper RH, van de Weijer T, Goossens GH, et al. Calorie restriction-like effects of 30 days of resveratrol supplementation on energy metabolism and metabolic profile in obese humans. Cell Metab 2011;14:612-22.

22. Morselli E, Marino G, Bennetzen MV, Eisenberg T, Megalou E, Schroeder S, et al. Spermidine and resveratrol induce autophagy by distinct pathways converging on the acetylproteome. J Cell Biol 2011;192:615-29.

23. Gonzales AM, Orlando RA. Curcumin and resveratrol inhibit nuclear factor-kappaB-mediated cytokine expression in adipocytes. Nutr Metab (Lond) 2008;5:17.

24. Barger JL, Kayo T, Vann JM, Arias EB, Wang J, Hacker TA, et al. A low dose of dietary resveratrol partially mimics caloric restriction and retards aging parameters in mice. PLoS One 2008; 3:e2264.

25. Kundu JK, Surh YJ. Cancer chemopreventive and therapeutic potential of resveratrol: mechanistic perspectives. Cancer Lett 2008;269:243-61.

26. Valenzano DR, Terzibasi E, Genade T, Cattaneo A, Domenici L, Cellerino A. Resveratrol prolongs lifespan and retards the onset of age-related markers in a short-lived vertebrate. Curr Biol 2006;16:296-300.

27. Chandrashekara KT, Shakarad MN. Aloe vera or resveratrol supplementation in larval diet delays adult aging in the fruit fly, Drosophila melanogaster. J Gerontol A Biol Sci Med Sci 2011;66: 965-71.

28. Choi KM, Lee HL, Kwon YY, Kang MS, Lee SK, Lee CK. Enhancement of mitochondrial function correlates with the extension of lifespan by caloric restriction and caloric restriction mimetics in yeast. Biochem Biophys Res Commun 2013;441:23642.

29. Szkudelska K, Szkudelski T. Resveratrol, obesity and diabetes. Eur J Pharmacol 2010;635:1-8.

30. Baron S, Bedarida T, Cottart CH, Vibert F, Vessieres E, Ayer A, et al. Dual effects of resveratrol on arterial damage induced by insulin resistance in aged mice. J Gerontol A Biol Sci Med Sci 2014; 69:260-9.

31. Pollack RM, Barzilai N, Anghel V, Kulkarni AS, Golden A, O'Broin P, et al. Resveratrol improves vascular function and mitochondrial number but not glucose metabolism in older adults. J Gerontol A Biol Sci Med Sci 2017;72:1703-9.

32. de Ligt M, Bruls YM, Hansen J, Habets MF, Havekes B, Nascimento EB, et al. Resveratrol improves ex vivo mitochondrial function but does not affect insulin sensitivity or brown adipose tissue in first degree relatives of patients with type 2 diabetes. Mol Metab 2018;12:39-47.

33. Minor RK, Baur JA, Gomes AP, Ward TM, Csiszar A, Mercken EM, et al. SRT1720 improves survival and healthspan of obese mice. Sci Rep 2011;1:70.

34. Mitchell SJ, Martin-Montalvo A, Mercken EM, Palacios HH, Ward TM, Abulwerdi G, et al. The SIRT1 activator SRT1720 extends lifespan and improves health of mice fed a standard diet. Cell Rep 2014;6:836-43.

35. Mercken EM, Mitchell SJ, Martin-Montalvo A, Minor RK, Almeida M, Gomes AP, et al. SRT2104 extends survival of male mice on a standard diet and preserves bone and muscle mass. Aging Cell 2014;13:787-96.

36. Guan R, Cai Z, Wang J, Ding M, Li Z, Xu J, et al. Hydrogen sulfide attenuates mitochondrial dysfunction-induced cellular senescence and apoptosis in alveolar epithelial cells by upregulating sirtuin 1. Aging (Albany NY) 2019;11:11844-64.

37. Diaz-Ruiz A, Lanasa M, Garcia J, Mora H, Fan F, Martin-Montalvo A, et al. Overexpression of CYB5R3 and NQO1, two NA- 
D+-producing enzymes, mimics aspects of caloric restriction. Aging Cell 2018;17:e12767.

38. Zhang H, Ryu D, Wu Y, Gariani K, Wang X, Luan P, et al. NAD+ repletion improves mitochondrial and stem cell function and enhances life span in mice. Science 2016;352:1436-43.

39. Martens CR, Denman BA, Mazzo MR, Armstrong ML, Reisdorph N, McQueen MB, et al. Chronic nicotinamide riboside supplementation is well-tolerated and elevates $\mathrm{NAD}+$ in healthy middle-aged and older adults. Nat Commun 2018;9:1286.

40. Elhassan YS, Kluckova K, Fletcher RS, Schmidt MS, Garten A, Doig CL, et al. Nicotinamide riboside augments the aged human skeletal muscle $\mathrm{NAD}+$ metabolome and induces transcriptomic and anti-inflammatory signatures. Cell Rep 2019;28:1717-1728. e6.

41. Mitchell SJ, Bernier M, Aon MA, Cortassa S, Kim EY, Fang EF, et al. Nicotinamide improves aspects of healthspan, but not lifespan, in mice. Cell Metab 2018;27:667-676. e4.

42. Yoshino J, Mills KF, Yoon MJ, Imai S. Nicotinamide mononucleotide, a key $\mathrm{NAD}(+)$ intermediate, treats the pathophysiology of diet- and age-induced diabetes in mice. Cell Metab 2011;14: 528-36.

43. Yang NC, Cho YH, Lee I. The lifespan extension ability of nicotinic acid depends on whether the intracellular NAD+ level is lower than the sirtuin-saturating concentrations. Int J Mol Sci 2019;21:142.

44. Zhang H, Tsao R. Dietary polyphenols, oxidative stress and antioxidant and anti-inflammatory effects. Curr Opin Food Sci 2016;8:33-42.

45. Pietrocola F, Marino G, Lissa D, Vacchelli E, Malik SA, Niso-Santano M, et al. Pro-autophagic polyphenols reduce the acetylation of cytoplasmic proteins. Cell Cycle 2012;11:3851-60.

46. Hung CH, Chan SH, Chu PM, Tsai KL. Quercetin is a potent anti-atherosclerotic compound by activation of SIRT1 signaling under oxLDL stimulation. Mol Nutr Food Res 2015;59:1905-17.

47. Chuang CC, Martinez K, Xie G, Kennedy A, Bumrungpert A, Overman A, et al. Quercetin is equally or more effective than resveratrol in attenuating tumor necrosis factor-\{alpha\}-mediated inflammation and insulin resistance in primary human adipocytes. Am J Clin Nutr 2010;92:1511-21.

48. Dower JI, Geleijnse JM, Gijsbers L, Zock PL, Kromhout D, HolIman PC. Effects of the pure flavonoids epicatechin and quercetin on vascular function and cardiometabolic health: a randomized, double-blind, placebo-controlled, crossover trial. Am J Clin Nutr 2015;101:914-21.

49. Alugoju P, Janardhanshetty SS, Subaramanian S, Periyasamy L, Dyavaiah M. Quercetin protects yeast saccharomyces cerevisiae pep4 mutant from oxidative and apoptotic stress and extends chronological lifespan. Curr Microbiol 2018;75:519-30.

50. Saul N, Pietsch K, Menzel R, Steinberg CE. Quercetin-mediated longevity in Caenorhabditis elegans: is DAF-16 involved? Mech Ageing Dev 2008;129:611-3.

51. Proshkina E, Lashmanova E, Dobrovolskaya E, Zemskaya N, Kudryavtseva A, Shaposhnikov M, et al. Geroprotective and radioprotective activity of quercetin, (-)-epicatechin, and ibuprofen in Drosophila melanogaster. Front Pharmacol 2016;7:505.

52. Spindler SR, Mote PL, Flegal JM, Teter B. Influence on longevity of blueberry, cinnamon, green and black tea, pomegranate, sesame, curcumin, morin, pycnogenol, quercetin, and taxifolin fed iso-calorically to long-lived, F1 hybrid mice. Rejuvenation Res 2013;16:143-51.

53. Zhu Y, Tchkonia T, Pirtskhalava T, Gower AC, Ding H, Giorgadze N, et al. The Achilles' heel of senescent cells: from transcriptome to senolytic drugs. Aging Cell 2015; 14:644-58.

54. Justice JN, Nambiar AM, Tchkonia T, LeBrasseur NK, Pascual R, Hashmi SK, et al. Senolytics in idiopathic pulmonary fibrosis: Results from a first-in-human, open-label, pilot study. EBioMedicine 2019;40:554-63.

55. Mu HN, Li Q, Pan CS, Liu YY, Yan L, Hu BH, et al. Caffeic acid attenuates rat liver reperfusion injury through sirtuin 3-dependent regulation of mitochondrial respiratory chain. Free Radic Biol Med 2015;85:237-49.

56. Tyszka-Czochara M, Konieczny P, Majka M. Caffeic acid expands anti-tumor effect of metformin in human metastatic cervical carcinoma HTB-34 cells: implications of AMPK activation and impairment of fatty acids de novo biosynthesis. Int J Mol Sci 2017;18:462.

57. Chiang EP, Tsai SY, Kuo YH, Pai MH, Chiu HL, Rodriguez RL, et al. Caffeic acid derivatives inhibit the growth of colon cancer: involvement of the PI3-K/Akt and AMPK signaling pathways. PLoS One 2014;9:e99631.

58. Tyszka-Czochara M, Bukowska-Strakova K, Kocemba-Pilarczyk KA, Majka M. Caffeic acid targets AMPK signaling and regulates tricarboxylic acid cycle anaplerosis while metformin downregulates HIF-1 $\alpha$-induced glycolytic enzymes in human cervical squamous cell carcinoma lines. Nutrients 2018;10:841.

59. Fontanilla CV, Wei X, Zhao L, Johnstone B, Pascuzzi RM, Farlow MR, et al. Caffeic acid phenethyl ester extends survival of a mouse model of amyotrophic lateral sclerosis. Neuroscience 2012;205:185-93.

60. Pietsch K, Saul N, Chakrabarti S, Sturzenbaum SR, Menzel R, Steinberg CE. Hormetins, antioxidants and prooxidants: defining quercetin-, caffeic acid- and rosmarinic acid-mediated life extension in C. elegans. Biogerontology 2011;12:329-47.

61. Havermann S, Chovolou Y, Humpf HU, Watjen W. Caffeic acid 
phenethylester increases stress resistance and enhances lifespan in Caenorhabditis elegans by modulation of the insulin-like DAF-16 signalling pathway. PLoS One 2014;9:e100256.

62. Li JQ, Fang JS, Qin XM, Gao L. Metabolomics profiling reveals the mechanism of caffeic acid in extending lifespan in Drosophila melanogaster. Food Funct 2020;11:8202-13.

63. Xiao K, Jiang J, Guan C, Dong C, Wang G, Bai L, et al. Curcumin induces autophagy via activating the AMPK signaling pathway in lung adenocarcinoma cells. J Pharmacol Sci 2013;123:102-9.

64. Wang C, Zhang X, Teng Z, Zhang T, Li Y. Downregulation of $\mathrm{PI} 3 \mathrm{~K} / \mathrm{Akt} / \mathrm{m}$ TOR signaling pathway in curcumin-induced autophagy in APP/PS1 double transgenic mice. Eur J Pharmacol 2014;740:312-20.

65. Na LX, Zhang YL, Li Y, Liu LY, Li R, Kong T, et al. Curcumin improves insulin resistance in skeletal muscle of rats. Nutr Metab Cardiovasc Dis 2011;21:526-33.

66. Suckow BK, Suckow MA. Lifespan extension by the antioxidant curcumin in Drosophila melanogaster. Int J Biomed Sci 2006; 2:402-5.

67. Kitani K, Osawa T, Yokozawa T. The effects of tetrahydrocurcumin and green tea polyphenol on the survival of male C57BL/ 6 mice. Biogerontology 2007;8:567-73.

68. Xiang L, Nakamura Y, Lim YM, Yamasaki Y, Kurokawa-Nose Y, Maruyama W, et al. Tetrahydrocurcumin extends life span and inhibits the oxidative stress response by regulating the FOXO forkhead transcription factor. Aging (Albany NY) 2011;3:1098109.

69. Murillo Ortiz BO, Fuentes Preciado AR, Ramirez Emiliano J, Martinez Garza S, Ramos Rodriguez E, de Alba Macias LA. Recovery of bone and muscle mass in patients with chronic kidney disease and iron overload on hemodialysis and taking combined supplementation with curcumin and resveratrol. Clin Interv Aging 2019; 14:2055-62.

70. Santos-Parker JR, Strahler TR, Bassett CJ, Bispham NZ, Chonchol MB, Seals DR. Curcumin supplementation improves vascular endothelial function in healthy middle-aged and older adults by increasing nitric oxide bioavailability and reducing oxidative stress. Aging (Albany NY) 2017;9:187-208.

71. Kasdallah-Grissa A, Mornagui B, Aouani E, Hammami M, El May M, Gharbi N, et al. Resveratrol, a red wine polyphenol, attenuates ethanol-induced oxidative stress in rat liver. Life Sci 2007;80:1033-9.

72. Abarikwu SO, Pant AB, Farombi EO. Quercetin decreases steroidogenic enzyme activity, NF- $\kappa \mathrm{B}$ expression, and oxidative stress in cultured Leydig cells exposed to atrazine. Mol Cell Biochem 2013;373:19-28.

73. Sahin A, Kursat Cingu A, Kaya S, Turkcu G, Ari S, Evliyaoglu O, et al. The protective effects of caffeic acid phenethyl ester in isoniazid and ethambutol-induced ocular toxicity of rats. Cutan Ocul Toxicol 2013;32:228-33.

74. Jena S, Chainy GB, Dandapat J. Expression of antioxidant genes in renal cortex of PTU-induced hypothyroid rats: effect of vitamin E and curcumin. Mol Biol Rep 2012;39:1193-203.

75. Qiu X, Brown K, Hirschey MD, Verdin E, Chen D. Calorie restriction reduces oxidative stress by SIRT3-mediated SOD2 activation. Cell Metab 2010;12:662-7.

76. Kailasam M, Kaneko G, Oo AK, Ozaki Y, Thirunavukkarasu AR, Watabe S. Effects of calorie restriction on the expression of manganese superoxide dismutase and catalase under oxidative stress conditions in the rotifer Brachionus plicatilis. Fish Sci 2011; 77:403-9.

77. Stankovic M, Mladenovic D, Ninkovic M, Vucevic D, Tomasevic T, Radosavljevic T. Effects of caloric restriction on oxidative stress parameters. Gen Physiol Biophys 2013;32:277-83.

78. Johansson M, Chen X, Milanova S, Santos C, Petranovic D. PUFA-induced cell death is mediated by Ycalp-dependent and -independent pathways, and is reduced by vitamin $\mathrm{C}$ in yeast. FEMS Yeast Res 2016;16:fow007.

79. Chen Q, Espey MG, Sun AY, Pooput C, Kirk KL, Krishna MC, et al. Pharmacologic doses of ascorbate act as a prooxidant and decrease growth of aggressive tumor xenografts in mice. Proc Natl Acad Sci U S A 2008;105:11105-9.

80. Carmona-Gutierrez D, Zimmermann A, Kainz K, Pietrocola F, Chen G, Maglioni S, et al. The flavonoid 4,4'-dimethoxychalcone promotes autophagy-dependent longevity across species. Nat Commun 2019;10:651.

81. Ayyadevara S, Bharill P, Dandapat A, Hu C, Khaidakov M, Mitra $S$, et al. Aspirin inhibits oxidant stress, reduces age-associated functional declines, and extends lifespan of Caenorhabditis elegans. Antioxid Redox Signal 2013;18:481-90.

82. Song C, Zhu C, Wu Q, Qi J, Gao Y, Zhang Z, et al. Metabolome analysis of effect of aspirin on Drosophila lifespan extension. Exp Gerontol 2017;95:54-62.

83. Strong R, Miller RA, Astle CM, Floyd RA, Flurkey K, Hensley $\mathrm{KL}$, et al. Nordihydroguaiaretic acid and aspirin increase lifespan of genetically heterogeneous male mice. Aging Cell 2008; 7:64150.

84. Wan QL, Zheng SQ, Wu GS, Luo HR. Aspirin extends the lifespan of Caenorhabditis elegans via AMPK and DAF-16/FOXO in dietary restriction pathway. Exp Gerontol 2013;48:499-506.

85. Zhao Q, Wang Z, Wang Z, Wu L, Zhang W. Aspirin may inhibit angiogenesis and induce autophagy by inhibiting mTOR signaling pathway in murine hepatocarcinoma and sarcoma models. Oncol Lett 2016;12:2804-10. 
86. ISIS-2 (Second International Study of Infarct Survival) Collaborative Group. Randomised trial of intravenous streptokinase, oral aspirin, both, or neither among 17,187 cases of suspected acute myocardial infarction: ISIS-2. Lancet 1988;2:349-60.

87. Ittaman SV, VanWormer JJ, Rezkalla SH. The role of aspirin in the prevention of cardiovascular disease. Clin Med Res 2014; 12:147-54.

88. McNeil JJ, Wolfe R, Woods RL, Tonkin AM, Donnan GA, Nelson $\mathrm{MR}$, et al. Effect of aspirin on cardiovascular events and bleeding in the healthy elderly. N Engl J Med 2018;379:150918.

89. McNeil JJ, Nelson MR, Woods RL, Lockery JE, Wolfe R, Reid $\mathrm{CM}$, et al. Effect of aspirin on all-cause mortality in the healthy elderly. N Engl J Med 2018;379:1519-28.

90. Gelbenegger G, Postula M, Pecen L, Halvorsen S, Lesiak M, Schoergenhofer C, et al. Aspirin for primary prevention of cardiovascular disease: a meta-analysis with a particular focus on subgroups. BMC Med 2019;17:198.

91. Eisenberg T, Abdellatif M, Schroeder S, Primessnig U, Stekovic $\mathrm{S}$, Pendl T, et al. Cardioprotection and lifespan extension by the natural polyamine spermidine. Nat Med 2016;22:1428-38.

92. Tirupathi Pichiah PB, Suriyakalaa U, Kamalakkannan S, Kokilavani P, Kalaiselvi S, SankarGanesh D, et al. Spermidine may decrease ER stress in pancreatic beta cells and may reduce apoptosis via activating AMPK dependent autophagy pathway. Med Hypotheses 2011;77:677-9.

93. Schwarz C, Stekovic S, Wirth M, Benson G, Royer P, Sigrist SJ, et al. Safety and tolerability of spermidine supplementation in mice and older adults with subjective cognitive decline. Aging (Albany NY) 2018;10:19-33.

94. Wirth M, Benson G, Schwarz C, Kobe T, Grittner U, Schmitz $D$, et al. The effect of spermidine on memory performance in older adults at risk for dementia: a randomized controlled trial. Cortex 2018;109:181-8.

95. Song YM, Lee YH, Kim JW, Ham DS, Kang ES, Cha BS, et al. Metformin alleviates hepatosteatosis by restoring SIRT1-mediated autophagy induction via an AMP-activated protein kinase-independent pathway. Autophagy 2015;11:46-59.

96. Shi WY, Xiao D, Wang L, Dong LH, Yan ZX, Shen ZX, et al. Therapeutic metformin/AMPK activation blocked lymphoma cell growth via inhibition of mTOR pathway and induction of autophagy. Cell Death Dis 2012;3:e275.

97. Martin-Montalvo A, Mercken EM, Mitchell SJ, Palacios HH, Mote PL, Scheibye-Knudsen M, et al. Metformin improves healthspan and lifespan in mice. Nat Commun 2013;4:2192.

98. Kazi RS, Banarjee RM, Deshmukh AB, Patil GV, Jagadeeshaprasad MG, Kulkarni MJ. Glycation inhibitors extend yeast chronological lifespan by reducing advanced glycation end products and by back regulation of proteins involved in mitochondrial respiration. J Proteomics 2017;156:104-12.

99. Onken B, Driscoll M. Metformin induces a dietary restriction-like state and the oxidative stress response to extend C. elegans Healthspan via AMPK, LKB1, and SKN-1. PLoS One 2010;5:e8758.

100. Cameron AR, Morrison VL, Levin D, Mohan M, Forteath C, Beall C, et al. Anti-inflammatory effects of metformin irrespective of diabetes status. Circ Res 2016;119:652-65.

101. Kim MY, Kim YS, Kim M, Choi MY, Roh GS, Lee DH, et al. Metformin inhibits cervical cancer cell proliferation via decreased AMPK O-GlcNAcylation. Anim Cells Syst (Seoul) 2019;23:302-9.

102. Nair V, Sreevalsan S, Basha R, Abdelrahim M, Abudayyeh A, Rodrigues Hoffman A, et al. Mechanism of metformin-dependent inhibition of mammalian target of rapamycin (mTOR) and Ras activity in pancreatic cancer: role of specificity protein (Sp) transcription factors. J Biol Chem 2014;289:27692-701.

103. Schmelzle T, Hall MN. TOR, a central controller of cell growth. Cell 2000;103:253-62.

104. Bjedov I, Toivonen JM, Kerr F, Slack C, Jacobson J, Foley A, et al. Mechanisms of life span extension by rapamycin in the fruit fly Drosophila melanogaster. Cell Metab 2010;11:35-46.

105. Harrison DE, Strong R, Sharp ZD, Nelson JF, Astle CM, Flurkey K, et al. Rapamycin fed late in life extends lifespan in genetically heterogeneous mice. Nature 2009;460:392-5.

106. Miller RA, Harrison DE, Astle CM, Fernandez E, Flurkey K, Han M, et al. Rapamycin-mediated lifespan increase in mice is dose and sex dependent and metabolically distinct from dietary restriction. Aging Cell 2014;13:468-77.

107. Choi KM, Hong SJ, van Deursen JM, Kim S, Kim KH, Lee CK. Caloric restriction and rapamycin differentially alter energy metabolism in yeast. J Gerontol A Biol Sci Med Sci 2017;73:29-38.

108. Dikicioglu D, Dereli Eke E, Eraslan S, Oliver SG, Kirdar B. Saccharomyces cerevisiae adapted to grow in the presence of lowdose rapamycin exhibit altered amino acid metabolism. Cell Commun Signal 2018; 16:85.

109. Kawahara T, Asthana S, Kneteman NM. m-TOR inhibitors: what role in liver transplantation? J Hepatol 2011;55:1441-51.

110. Sataranatarajan K, Ikeno Y, Bokov A, Feliers D, Yalamanchili H, Lee HJ, et al. Rapamycin increases mortality in $\mathrm{db} / \mathrm{db}$ mice, a mouse model of type 2 diabetes. J Gerontol A Biol Sci Med Sci 2016;71:850-7.

111. Lamming DW, Ye L, Katajisto P, Goncalves MD, Saitoh M, Stevens DM, et al. Rapamycin-induced insulin resistance is medi- 
ated by mTORC2 loss and uncoupled from longevity. Science 2012;335:1638-43.

112. Lamming DW, Ye L, Sabatini DM, Baur JA. Rapalogs and mTOR inhibitors as anti-aging therapeutics. J Clin Invest 2013;123:980-9.

113. Leontieva OV, Blagosklonny MV. Gerosuppression by panmTOR inhibitors. Aging (Albany NY) 2016;8:3535-51.

114. Shavlakadze T, Zhu J, Wang S, Zhou W, Morin B, Egerman MA, et al. Short-term low-dose mTORC1 inhibition in aged rats counter-regulates age-related gene changes and blocks age-related kidney pathology.J Gerontol A Biol Sci Med Sci 2018;73: 845-52.

115. Cassano T, Magini A, Giovagnoli S, Polchi A, Calcagnini S, Pace $\mathrm{L}$, et al. Early intrathecal infusion of everolimus restores cognitive function and mood in a murine model of Alzheimer's disease. Exp Neurol 2019;311:88-105.

116. Baker DJ, Childs BG, Durik M, Wijers ME, Sieben CJ, Zhong J, et al. Naturally occurring p16(Ink4a)-positive cells shorten healthy lifespan. Nature 2016;530:184-9.

117. Fuhrmann-Stroissnigg H, Ling YY, Zhao J, McGowan SJ, Zhu Y, Brooks RW, et al. Identification of HSP90 inhibitors as a novel class of senolytics. Nat Commun 2017;8:422.

118. Xu M, Pirtskhalava T, Farr JN, Weigand BM, Palmer AK,
Weivoda MM, et al. Senolytics improve physical function and increase lifespan in old age. Nat Med 2018;24:1246-56.

119. Roberts MN, Wallace MA, Tomilov AA, Zhou Z, Marcotte GR, Tran D, et al. A ketogenic diet extends longevity and healthspan in adult mice. Cell Metab 2017;26:539-546. e5.

120. Douris N, Melman T, Pecherer JM, Pissios P, Flier JS, Cantley LC, et al. Adaptive changes in amino acid metabolism permit normal longevity in mice consuming a low-carbohydrate ketogenic diet. Biochim Biophys Acta 2015;1852(10 Pt A): 205665.

121. Bueno NB, de Melo IS, de Oliveira SL, da Rocha Ataide T. Very-low-carbohydrate ketogenic diet v. low-fat diet for longterm weight loss: a meta-analysis of randomised controlled trials. Br J Nutr 2013;110:1178-87.

122. Malavolta M, Pierpaoli E, Giacconi R, Costarelli L, Piacenza F, Basso A, et al. pleiotropic effects of tocotrienols and quercetin on cellular senescence: introducing the perspective of senolytic effects of phytochemicals. Curr Drug Targets 2016;17:447-59.

123. Desjardins D, Cacho-Valadez B, Liu JL, Wang Y, Yee C, Bernard $\mathrm{K}$, et al. Antioxidants reveal an inverted U-shaped dose-response relationship between reactive oxygen species levels and the rate of aging in Caenorhabditis elegans. Aging Cell 2017; 16:104-12. 